\title{
An Analytical Study of Authentication and Access Control Issues in Cloud Computing
}

\author{
Dr. Nilesh Mahajan \\ Prof. Bharti Vidyapeeth Deemed \\ University, IMED Pune
}

Mrs. Devyani Patil.

Asst. Prof. Arihant College

Camp, Pune

\begin{abstract}
-
Cloud Computing is on demand network. It is a network architecture where you can store, retrieve and access data at anytime and anywhere if you are a legitimate user of Cloud environment. In simple client server architecture whatever it is in limited scope. Second question is of economically it is not affordable to maintain it if big data is stored on this architecture. Cloud computing provides you space for your data on rent and giving you a very good security. In Cloud computing Authentication the important term is user authentication. At the time of entering in the cloud computing environment which type authentication user is facing that is important for secured cloud computing environment. This paper is focusing on problems faced by user related to authentication and access control challenges in cloud computing environment.
\end{abstract}

Keywords-

Cloud Computing, Authentication, Access Control, Virtual Environment.

\section{Introduction-}

Cloud Computing is new technology in the software sector. Nowadays all IT companies are switching to cloud computing environment. Cloud Computing is type of distributed computing. In this architecture a specific application may run on different linked computers simultaneously. [2] This provides facility like mass storage, low storage cost, complex calculations with ease and ease of access etc. Data stored on cloud computing is safe. But nowadays cloud user's facing problems in authentication and access control. There are many methods available in the market for authentication to the cloud environment. Now the difference is in between which methods are used in the client-server architecture and which are used in the cloud computing environment. [4]

\section{Attacks happens easily with user.}

Some such issues are listed below:

a) Password Guessing Attack: This is simple technique of attack in which password is guessed by a person who know you.

b) Replay Attack: This attack tracks authenticated user's packet and create fake information for the unauthorized users.

c) Man-in-the-middle Attack: In this case attacker attitude is like a user and try to acquire password from the server.

d) Masquerade Attack: The attacker pretend like a verifier and capture authentication keys from the authenticated user.

e) Insider Attack: Attacker intentionally steals the private information of the authenticated user. 
f) Phishing Attack: Social Engineering sites such as fake emails, fake SMS and phone calls to demand the user expose his password or authentication keys. [1][5][]9]

\section{Survey taken to find out issues related to authentication nd access control challenges.}

To find out the issues related to user authentication some questions are asked to the peoples who is working on cloud environment. Throughout the survey it is observed that some user are satisfied with security but some are getting frustrated due to increasing levels of authentication. User is ok with the two or three level authentication but if it more than that then he thought that it is a boring process. User is ok when he want to use the cloud environment more than 2 or 3 hours, but if user want to use cloud environment just for less than one hour, he is getting frustrated. Total data of 87 users is collected from different IT companies.[7] Response got from the users shows that some user get frustrated in two step authentication, some are in three and some are in four. Few user's opinion is for the email accounts two step verification is ok but for the remaining like office account want to activate from clod computing is not secure with the two step verification. Figure a is showing about that how users are agree and disagree with all over authentication.

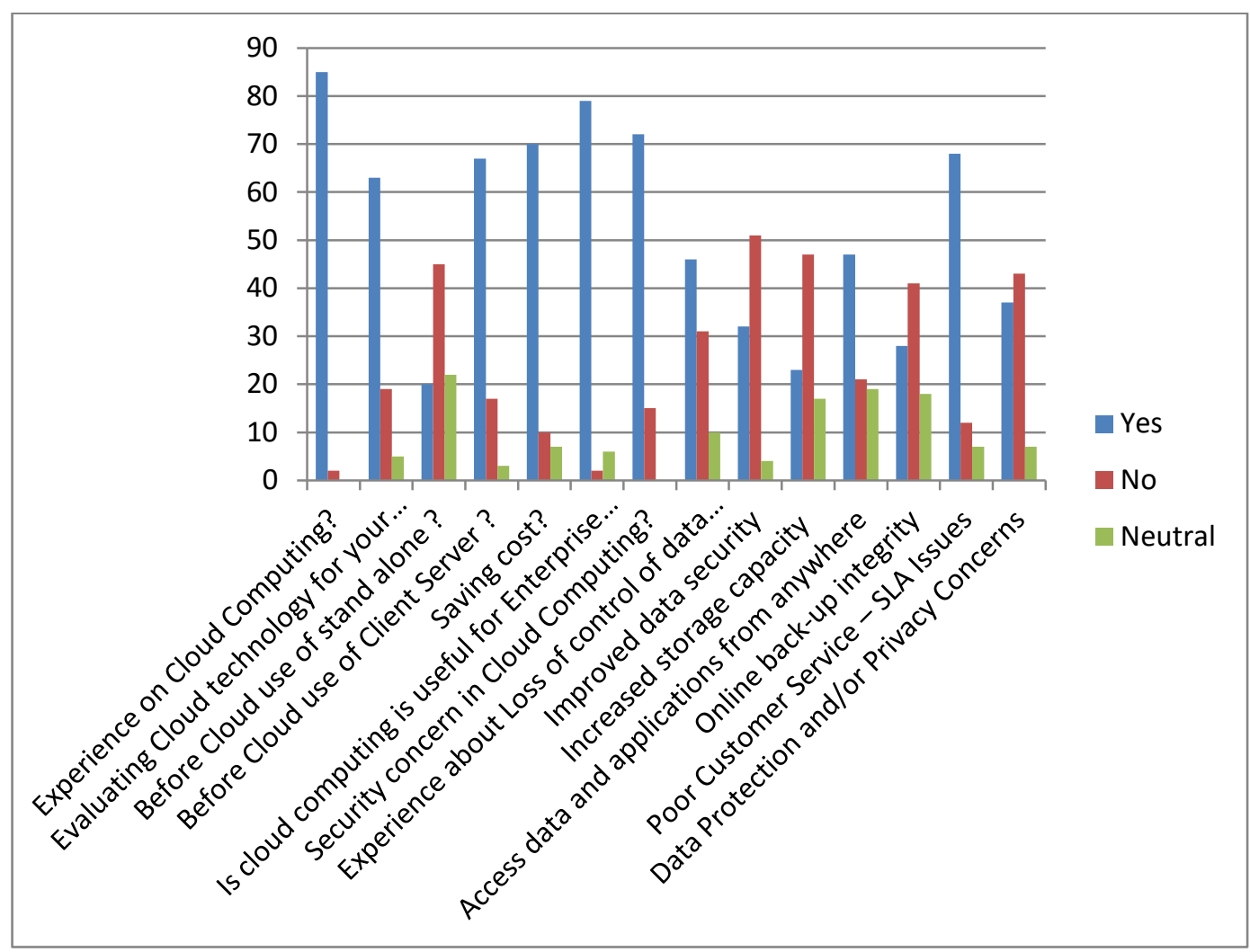

Fig. a Analysis about security threats observed by user.

Questions asked for this analysis were related to the all over cloud environment. It asks about experience about cloud computing in ERP, Loss of Control data, Online backup Integrity, Poor SLA, Increased storage capacity etc.

III. Analysis about user's opinion on security stages.

In this security stages study of all security stages is concerned. This graph analysis shows users views on two stage, three stage, four stage and five stage authentication. Fig.b shows this analysis of user's view against authentication levels. In first level authentication it shows 
DOI: https://dx.doi.org/10.26808/rs.ca.i8v2.13 International Journal of Computer Application (2250-1797) Issue 8 Volume 2, March-April 2018

only user id and password.[9] But extra terms added in next levels as OTP is added in three stage, text Captcha is added in four stage authentication, Sound captcha is added in Five Stage Authentication also Biometric is added in Six stage authentication.

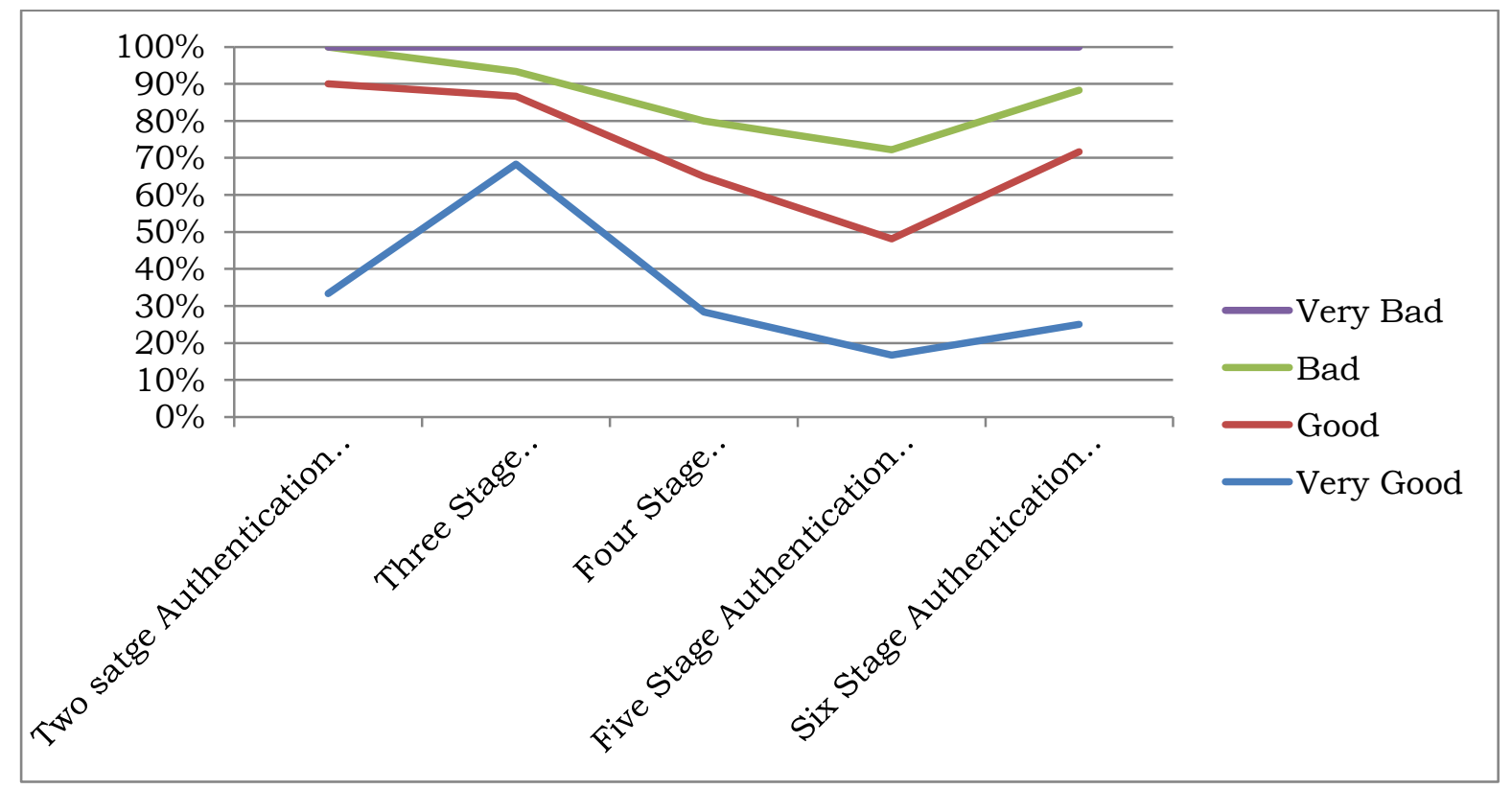

Fig. b User's view against authentication stages.

\section{Conclusion-}

All over paper is discussing the authentication and access control challenges over the cloud computing environment. As if there are problems arises in the previously available methods my study suggesting a new study of creation of certificate credentials. In this process when user is passing three stage of authentication then through a system generated certificate credential will be check it out that is entered user is authenticated or not.

\section{References:}

1)"Security Architecture of Cloud Computing", V.KRISHNA REDDY 1, Dr. L.S.S.REDDY, International Journal of Engineering Science and Technology (!JEST), Vol. 3 No. 9 September 2011
2)IBM.IBM
Cloud
Computing
Center
[OL].
[2009-0S-0S]. http://www.900.ibm.comlibm/ideasfromibmlcn/cloud/index.shtml.

3)Balachandra, P. V. Ramakrishna and A. Rakshit "Cloud Security Issues",IEEE International Conference on Services Computing Security Issues,pp. 517-520, 2009.

4)Muthunagai, Karthic and SujathaIEEE, Virtualization Techniques pp.174-17, 2012.

5)D. W. Chadwick, M. Casenove. "Security APIs for My Private Cloud:Granting access to anyone, from anywhere at any time." 2011 IEEE 3rd International Conference on Cloud Computing Technology and Science,2011, p.792-798.

6)Amazon Web Services, http://aws.amazon.com.

7)Cloud computing security. URL :http://en.wikipedia.org/wiki/Cloud_comput ing_security. 8)J. Zheng, T. Ng, K. Sripanidkulchai, and Z. Liu, "Pacer: A progress management system for live virtual machine migration in cloud computing," IEEE Transactions on Network and Service Management, vol. 10, no. 4, pp. 369-382, 2013

9)“Cloud guide,” Cloud Security Alliance.December 2009. 